\title{
Clinical Outcomes of Critically III Patients with COVID-19 by Race
}

\author{
Fahad Marmarchi ${ }^{1} \cdot$ Michael Liu $^{1} \cdot$ Srikant Rangaraju $^{1} \cdot$ Sara C. Auld ${ }^{2,3} \cdot$ Maria Christina Creel-Bulos $^{4}$. \\ Christine L Kempton ${ }^{5} \cdot$ Milad Sharifpour $^{4} \cdot$ Manila Gaddh $^{5} \cdot$ Roman Sniecinski $^{6} \cdot$ Cheryl L. Maier $^{7} \cdot$ Fadi Nahab $^{8} \cdot$ The \\ Emory COVID-19 Quality and Clinical Research Collaborative (QCRC)
}

Received: 25 August 2020 / Revised: 25 October 2020 / Accepted: 10 January 2021 / Published online: 19 January 2021

(C) W. Montague Cobb-NMA Health Institute 2021

\begin{abstract}
Background Studies of COVID-19 have shown that African Americans have been affected by the virus at a higher rate compared to other races. This cohort study investigated comorbidities and clinical outcomes by race among COVID-19 patients admitted to the intensive care unit.

Methods This is a case series of critically ill patients admitted with COVID-19 to an academic healthcare system in Atlanta, Georgia. The study included all critically ill hospitalized patients between March 6, 2020, and May 5, 2020. Clinical outcomes during hospitalization included mechanical ventilation, renal replacement therapy, and mortality stratified by race.

Results Of 288 patients included (mean age, $63 \pm 16$ years; $45 \%$ female), 210 (73\%) were African American. African Americans had significantly higher rates of comorbidities compared to other races, including hypertension $(80 \% \mathrm{vs} 59 \%, P=0.001)$, diabetes $(49 \%$ vs $34 \%, P=0.026)$, and mean BMI $\left(33 \mathrm{~kg} / \mathrm{m}^{2}\right.$ vs $\left.28 \mathrm{~kg} / \mathrm{m}^{2}, P<0.001\right)$. Despite African Americans requiring continuous renal replacement therapy during hospitalization at higher rates than other races $(27 \%$ vs $13 \%, P=0.011)$, rates of intubation, intensive care unit length of stay, and overall mortality $(30 \%$ vs $24 \%, P=0.307)$ were similar.

Conclusion This racially diverse series of critically ill COVID-19 patients shows that despite higher rates of comorbidities at hospital admission in African Americans compared with other races, there was no significant difference in mortality.
\end{abstract}

Keywords COVID-19 $\cdot$ Mortality $\cdot$ Race $\cdot$ African American · Outcomes

Fadi Nahab

fnahab@emory.edu

1 Department of Neurology, Emory University School of Medicine, Atlanta, GA, USA

2 Emory Critical Care Center, Division of Pulmonary, Allergy, Critical Care, and Sleep Medicine, Department of Medicine, Emory University School of Medicine, Atlanta, GA, USA

3 Department of Epidemiology, Emory University Rollins School of Public Health, Atlanta, GA, USA

4 Department of Anesthesiology, Division of Critical Care Medicine, Emory University School of Medicine, Atlanta, GA, USA

5 Department of Hematology and Medical Oncology, Emory University School of Medicine, Atlanta, GA, USA

6 Department of Anesthesiology, Emory University School of Medicine, Atlanta, GA, USA

7 Department of Pathology and Laboratory Medicine, Emory University School of Medicine, Atlanta, GA, USA

8 Department of Neurology \& Pediatrics, Emory University, 1365 Clifton Road, Clinic B, Suite 2200, Atlanta, GA 30322, USA

\section{Introduction}

The coronavirus disease 2019 (COVID-19) has led to death tolls surpassing 2,000,000 people globally and 395,000 people in the USA $[1,2]$. Studies to date have shown that COVID-19 infects African Americans (AA) at a higher rate compared with other major races in the USA; the Centers for Disease Control and Prevention (CDC) has found that AA have a $178.1 / 100,000$ rate of COVID-19, second to only Native Americans and Native Alaskans $(221.2 / 100,000)$ and significantly higher than that of white Americans (40.1/ 100,000) [3-6]. The racial disparity seen with COVID-19 rates has been associated with differences in household size, social distancing, community education, and ability to work from home which some communities of color do not have [7, 8]. These social issues and healthcare disparities have been apparent in the past but have been further amplified by COVID-19 [7, 9]. In addition to social factors, studies have also shown that AA have high frequencies of hypertension, diabetes, chronic kidney disease, and obesity, factors associated with poor outcomes from COVID-19 [10-13]. 
There are limited data assessing racial differences in clinical outcomes of critically ill COVID-19 patients. Cohort studies in Louisiana and Georgia found that race was not associated with hospital mortality after adjusting for differences in sociodemographic and clinical characteristics $[6,14]$. The objective of our study was to evaluate whether racial differences impact the frequency of in-hospital complications and overall mortality of critically ill patients in a racially diverse cohort.

\section{Methods}

This observational cohort study includes data from hospitalized COVID-19 patients admitted from March 6, 2020, to May 5, 2020, who were admitted to Emory Healthcare, an academic healthcare system in Atlanta, Georgia. Patients 18 years or older that were hospitalized in the ICU were eligible for inclusion once positive COVID-19 status was confirmed based on a severe acute respiratory syndrome coronavirus 2 polymerase chain reaction assay. Other COVID-19positive patients who did not reach ICU level of care needs were excluded. Patient data was obtained from the electronic medical record (EMR) (Cerner Millennium EMR, Kansas City, MO, USA) using proprietary data collection software internally developed on the Oracle Apex platform (Redwood City, CA, USA). Categorical and continuous data elements were automatically extracted using an internally validated technique from the Cerner Millennium EMR through the Apex platform. Data elements contained in free text were manually abstracted by members of the Emory COVID-19 Quality and Clinical Research Collaborative (QCRC) and entered into the Apex platform [15]. The variables that were investigated included data on demographics, medical comorbidities, hospitalization, and outcomes. For analysis, data from the Apex environment were merged with data directly extracted from the Millennium platform to create a comprehensive dataset of all investigated variables.

Data were collected through June 9, 2020, and analyzed using a chi-square or independent sample $t$ tests for categorical and continuous variables, respectively, with a two-sided $P$ value of less than 0.05 considered statistically significant. Univariate analysis and multivariate analysis were done on all categorical and continuous variables on the outcome of mortality. Significance for univariate analysis was set at a $P$ value of less than 0.1 and for multivariate analysis significance was set at a $P$ value of less than 0.05 . All statistical analysis was SPSS version 25 (IBM Corporation, Armonk, New York). This study was approved by the Emory University Institutional Review Board.

\section{Results}

During the study period, 288 patients were admitted to the ICU with a diagnosis of COVID-19. The mean age was 63 $( \pm 16)$ years, $131(45 \%)$ were female, $209(73 \%)$ were AA, 68 (24\%) were white, 10 (3.4\%) were Asian, and 1 (0.3\%) was multiple race. Compared with non-AA, AAs had significantly higher rates of hypertension $(80 \%$ vs $59 \%, P=0.001)$ and diabetes $(49 \%$ vs $34 \%, P=0.026)$ and a higher mean BMI (33 vs $28, P<0.001$ ) (Table 1 ). There were no differences between races in history of coronary artery disease, stroke, chronic kidney disease, chronic obstructive pulmonary disease, asthma, obstructive sleep apnea, HIV, or other immunocompromised state.
Table 1 Comorbidities in ICU hospitalized patients by race

\begin{tabular}{lcccr}
\hline Admission characteristics & Total $(n=288)$ & AA $(n=209)$ & Other $(n=79)$ & $P$ value \\
\hline Age, SD & $63(16)$ & $62(15)$ & $65(18)$ & 0.269 \\
Female, $n(\%)$ & $131(45)$ & $95(45)$ & $36(46)$ & 0.986 \\
HTN, $n(\%)$ & $214(74)$ & $167(80)$ & $47(59)$ & $<0.001$ \\
DM, $n(\%)$ & $129(45)$ & $102(49)$ & $27(34)$ & 0.026 \\
BMI $(n=285)$ mean (SD) & $32(8)$ & $33(9)$ & $28(6)$ & $<0.001$ \\
CAD, $n(\%)$ & $40(14)$ & $25(12)$ & $15(19)$ & 0.124 \\
Stroke, $n(\%)$ & $39(14)$ & $26(12)$ & $13(16)$ & 0.374 \\
CKD, $n(\%)$ & $43(15)$ & $34(16)$ & $9(11)$ & 0.300 \\
COPD, $n(\%)$ & $19(7)$ & $12(6)$ & $7(9)$ & 0.341 \\
Asthma, $n(\%)$ & $28(10)$ & $17(8)$ & $11(14)$ & 0.139 \\
OSA, $n(\%)$ & $24(8)$ & $18(9)$ & $6(8)$ & 0.780 \\
HIV, $n(\%)$ & $5(2)$ & $5(2)$ & $0(0)$ & 0.328 \\
Other immunocompromised state, $n(\%)$ & $11(4)$ & $7(3)$ & $4(5)$ & 0.501 \\
\hline
\end{tabular}

HTN hypertension, $D M$ diabetes mellitus, $B M I$ body mass index, $C A D$ coronary artery disease, $C K D$ chronic kidney disease, COPD chronic obstructive pulmonary disease, OSA obstructive sleep apnea, HIV human immunodeficiency virus 
Table 2 Clinical outcomes in ICU patients by race

\begin{tabular}{lcccc}
\hline Hospitalization data and outcome & Total $(n=288)$ & AA $(n=209)$ & Other $(n=79)$ & $P$ value \\
\hline Hospitalization duration, mean days (SD) & $18(12)$ & $18(12)$ & $19(12)$ & 0.700 \\
ICU length, mean days (SD) & $11(9)$ & $10(8)$ & $11(9)$ & 0.330 \\
Intubated, $n(\%)$ & $212(74)$ & $155(74)$ & $57(72)$ & 0.730 \\
Intubation length, mean days (SD) & $11(8)$ & $11(8)$ & $11(9)$ & 0.952 \\
CRRT, $n(\%)$ & $66(23)$ & $56(27)$ & $10(13)$ & 0.011 \\
CRRT length, mean days (SD) & $9(6)$ & $9(6)$ & $9(7)$ & 0.937 \\
HD, $n(\%)$ & $39(14)$ & $32(15)$ & $7(9)$ & 0.153 \\
HD length, mean days (SD) & $7(5)$ & $7(5)$ & $6(6)$ & 0.727 \\
SOFA, mean (SD) & $6(4)$ & $6(4)$ & $7(4)$ & 0.215 \\
Death, $n(\%)$ & $82(29)$ & $63(30)$ & $19(24)$ & 0.307 \\
\hline
\end{tabular}

$I C U$ intensive care unit, $C R R T$ continuous renal replacement therapies, $H D$ hemodialysis, SOFA sequential organ failure assessment
Mean hospital length of stay was $18( \pm 12)$ days, and ICU length of stay was $11( \pm 9)$ days. Overall, $212(74 \%)$ patients were intubated for a mean of $11( \pm 8)$ days; $66(23 \%)$ patients required continuous renal replacement therapy (CRRT) for a mean of $9( \pm 6)$ days. There were $82(29 \%)$ patients who died. There was no significant difference in length of stay, intubation rates, length of intubation, and deaths between AAs and non-AA patients (Table 2). CRRT was more frequently required in AA patients $(27 \%$ vs $13 \%, P=0.011)$, whereas intermittent hemodialysis rates were similar $(15 \%$ vs $9 \%, P=$ $0.153)$.
In univariable analysis, factors associated with in-hospital mortality included age (OR: 1.058, 95\% CI: 1.036-1.080, $P$ value: <0.001), hypertension (OR: $1.793,95 \%$ CI: 0.949 3.387, $P$ value: 0.072 ), coronary artery disease (OR: 2.081 , 95\% CI: $1.046-4.140, P$ value: 0.037$)$, hospital length of stay (LOS) (OR: 0.918 95\% CI: $0.888-0.948, P$ value: $<0.001$ ), ICU LOS (OR: 0.872, 95\% CI: 0.941-1.004, $P$ value: 0.087 ), intubation rate (OR: $2.091,95 \%$ CI: $1.094-3.997, P$ value: 0.026), CRRT use (OR: $4.591,95 \%$ CI: $2.563-8.226, P$ value: $<0.001$ ), and BMI (OR: 0.909, 95\% CI: 0.871-0.949, $P$ value: $<0.001$ ), but not race (Table 3 ). In multivariable analysis,
Table 3 Variables associated with inpatient mortality in univariate analysis

\begin{tabular}{lllr}
\hline Variable & Odds ratio & $95 \%$ confidence interval & $P$ value \\
\hline Age & 1.058 & $1.036-1.080$ & $<0.001$ \\
Female & 0.914 & $0.546-1.530$ & 0.733 \\
African American & 1.363 & $0.752-2.469$ & 0.308 \\
HTN & 1.793 & $0.949-3.387$ & 0.072 \\
DM & 1.169 & $0.700-1.953$ & 0.551 \\
BMI & 0.909 & $0.871-0.949$ & $<0.001$ \\
CAD & 2.081 & $1.046-4.140$ & 0.037 \\
Stroke & 1.491 & $0.732-3.035$ & 0.271 \\
CKD & 1.607 & $0.814-3.172$ & 0.171 \\
COPD & 1.509 & $0.572-3.978$ & 0.406 \\
Asthma & 0.517 & $0.189-1.409$ & 0.197 \\
OSA & 0.477 & $0.158-1.441$ & 0.189 \\
HIV & 3.873 & $0.635-23.620$ & 0.142 \\
Other immunocompromised state & 0.547 & $0.116-2.589$ & 0.447 \\
Hospitalization duration & 0.918 & $0.888-0.948$ & $<0.001$ \\
ICU length of stay & 0.972 & $0.941-1.004$ & 0.087 \\
Intubated & 2.091 & $1.094-3.997$ & 0.026 \\
CRRT & 4.591 & $2.563-8.226$ & $<0.001$ \\
HD & 0.985 & $0.465-2.085$ & 0.968 \\
SOFA & 1.042 & $0.975-1.112$ & 0.224 \\
\hline
\end{tabular}


Table 4 Multivariable analysis of variables associated with in-hospital mortality

\begin{tabular}{lclr}
\hline Variable & Odds ratio & $95 \%$ confidence interval & $P$ value \\
\hline Age & 1.094 & $1.056-1.134$ & $<0.001$ \\
BMI & 0.886 & $0.825-0.951$ & 0.001 \\
HTN & 0.859 & $0.309-2.391$ & 0.771 \\
CAD & 2.486 & $0.907-6.811$ & 0.077 \\
Hospital duration & 0.760 & $0.697-0.828$ & $<0.001$ \\
ICU length of stay & 1.148 & $1.044-1.262$ & 0.004 \\
Intubation & 4.824 & $1.545-15.062$ & 0.007 \\
CRRT & 20.806 & $6.874-62.981$ & $<0.001$ \\
\hline
\end{tabular}

$B M I$ body mass index, $H T N$ hypertension, $C A D$ coronary artery disease, $I C U$ intensive care unit, $C R R T$ continuous renal replacement therapies

age (OR: 1.094, 95\% CI: 1.056-1.134, $P$ value: $<0.001$ ), hospital duration (OR: $0.760,95 \%$ CI: $0.697-0.828, P$ value: $<0.001$ ), ICU length of stay (OR: $1.148,95 \%$ CI: 1.044 $1.262, P$ value: 0.004$)$, intubation rate (OR: $4.824,95 \% \mathrm{CI}$ : 1.545-15.062, $P$ value: 0.007 ), CRRT use (OR: 20.806, 95\% CI: 6.874-62.981, $P$ value: $<0.001$ ), and BMI (OR: 0.886, 95\% CI: $0.825-0.951, P$ value: 0.001$)$ were associated with in-hospital mortality (Table 4).

\section{Discussion}

In our racially diverse cohort of critically ill COVID-19 patients including $73 \%$ AAs, we found that intubation rate and in-hospital mortality were similar between AA and non-AA patients. This was found despite AAs having higher rates of comorbidities including hypertension, diabetes, and obesity compared to non-AAs. AAs did have higher rates of CRRT than non-AAs despite having similar rates of chronic kidney disease history on admission. The prevalence of these comorbidities observed in AA vs non-AA has been reported previously and increases the complication rates in patients infected with COVID-19 [10-13, 16, 17]. These results are similar to those of a cohort study in Louisiana, which found similar inhospital mortality rates between AAs and non-AAs despite AAs having higher rates of acute renal failure [6]. In another cohort study from Georgia which included ICU and non-ICU patients, AA patients were found to have similar rates of invasive mechanical ventilation and death compared to non-AA patients [13].

Though COVID-19 proved to be challenging to all hospital systems, Atlanta did not observe the overwhelming case numbers early in the pandemic as some other major cities [1]. Our study was limited to metropolitan Atlanta hospitals within one academic health system in Georgia and may have limited generalizability to other healthcare settings. However, our study represents a large cohort of critically ill COVID-19 patients including a large proportion of AA patients. Additionally, this study is limited by being a retrospective analysis of an ICUspecific cohort that does not compare mortality rates outside of an ICU setting. Despite these limitations, our study shows similar in-hospital mortality rates between AAs and other races despite AAs having higher rates of comorbidities at admission and higher rates of RRT in the hospital. Whether these findings are due to pre-hospital social factors [4], differences in the disease manifestation or differences in provider treatments require further study.

\section{Conclusion}

Despite critically ill AA with COVID-19 presenting with higher rates of comorbidities compared to other races, rates of intubation and mortality were similar at an academic healthcare system in Atlanta, Georgia. Our findings show that in a healthcare system that has adequate critical care capacity to provide treatment to the sickest of COVID-19 patients, we were able to limit disparities in mortality despite the social factors that contribute to AAs presenting with higher rates of comorbid illnesses on admission.

Supplementary Information The online version contains supplementary material available at https://doi.org/10.1007/s40615-021-00966-0.

Funding This study was supported by Grant K23 AL134182 from the National Institute of Health/National Institute of Allergy and Infectious Disease. Sara C. Auld MD, MSc.

\section{Compliance with Ethical Standards}

Conflict of Interest The authors declare that they have no conflict of interest.

\section{References}

1. center JHUaMCVR. COVID-19 Dashboard by the Center for Systems Science and Engineering (CSSE) at Johns Hopkins University (JHU). 2020.

2. Zhang B, Zhou X, Qiu Y, Song Y, Feng F, Feng J, et al. Clinical characteristics of 82 cases of death from COVID-19. PLoS One. 2020;15(7): 0235458.

3. CenterOfDiseaseControl. Racial \& Ethnic Minority Groups 2020; https://www.cdc.gov/coronavirus/2019-ncov/need-extraprecautions/racial-ethnic-minorities.html.

4. Cowger TL, Davis BA, Etkins OS, Makofane K, Lawrence JA, Bassett MT, et al. Comparison of weighted and unweighted population data to assess inequities in coronavirus disease 2019 deaths by race/ethnicity reported by the US Centers for Disease Control and Prevention. JAMA Netw Open. 2020;3(7):e2016933.

5. Millett GA, Jones AT, Benkeser D, Baral S, Mercer L, Beyrer C, et al. Assessing differential impacts of COVID-19 on black communities. Ann Epidemiol. 2020;47:37-44. 
6. Price-Haywood EG, Burton J, Fort D, Seoane L. Hospitalization and mortality among black patients and white patients with Covid19. N Engl J Med. 2020;382(26):2534-43.

7. Yancy CW. COVID-19 and African Americans. JAMA. 2020;323(19):1891-2.

8. Duffin E. Race and ethnicity of U.S. households in 2015, by size. Statista. 2020.

9. Gold JAW, Rossen LM, Ahmad FB, Sutton P, Li Z, Salvatore PP, et al. Race, ethnicity, and age trends in persons who died from COVID-19 - United States, may-august 2020. MMWR Morb Mortal Wkly Rep. 2020;69(42):1517-21.

10. CenterOfDiseaseControl. African American Health 2017.

11. Cooper RS. Genetic factors in ethnic disparities in health. National Research Council (US) Panel on Race, Ethnicity, and Health in Later Life. 2004;8.

12. Health NIo. Why are some genetic conditions more common in particular ethnic groups? . Genetic Home refrence.
13. Palmer ND, McDonough CW, Hicks PJ, Roh BH, Wing MR, An SS, et al. A genome-wide association search for type 2 diabetes genes in African Americans. PLoS One. 2012;7(1):e29202.

14. Gold JAW, Wong KK, Szablewski CM, Patel PR, Rossow J, da Silva J, et al. Characteristics and clinical outcomes of adult patients hospitalized with COVID-19 - Georgia, march 2020. MMWR Morb Mortal Wkly Rep. 2020;69(18):545-50.

15. Auld S, Caridi-Scheible M, Blum JM, et al. ICU and ventilator mortality among critically ill adults with COVID-19. Society of Critical Care Medicine. 2020.

16. Guan WJ, Liang WH, Zhao Y, et al. Comorbidity and its impact on 1590 patients with COVID-19 in China: a nationwide analysis. Eur Respir J. 2020;55(5).

17. Sanyaolu A, Okorie C, Marinkovic A, et al. Comorbidity and its impact on patients with COVID-19. SN Compr Clin Med. 2020:1-8.

Publisher's Note Springer Nature remains neutral with regard to jurisdictional claims in published maps and institutional affiliations. 